\title{
A concept for human-cyber-physical systems of future wind turbines towards Industry 5.0
}

\author{
Xiao Chen ${ }^{1, *}$, Martin A. Eder ${ }^{1, *}$, ASM Shihavuddin ${ }^{2, *}$
}

1. Department of Wind Energy, Technical University of Denmark, Frederiksborgvej 399, 4000

Roskilde, Denmark

2. EEE Department, Green University of Bangladesh, 220/D, Begum Rokeya Sarani, Dhaka -1207, Bangladesh

X.C.: xiac@dtu.dk; M.A.E.: maed@dtu.dk; A.S.: shihav@eee.green.edu.bd

\begin{abstract}
:
This work proposes a novel concept for a semi-autonomous human-cyber-physical system (HCPS) to operate next-generation wind turbines on the way towards Industry 5.0. The exponential increase in the complexity of future wind turbines requires artificial intelligence (AI) to operate the machines efficiently and consistently. Evolving current Industry 4.0 digital twin technology beyond a sole aid for a human decision-making process, the digital twin in the proposed system is utilized for highly effective training of the AI through machine learning. Human intelligence (HI) is elevated to a supervisory level, making highlevel decisions through a human-machine interface to break the autonomy when needed. This paper points out a plausible way to realize the HCPS by identifying strategic development demands for the key enabling technologies projected from readily available knowledge.
\end{abstract}

Keywords: wind turbine; human intelligence; artificial intelligence; machine learning; digital twin

$\begin{array}{ll}\text { Nomenclature } & \\ \text { HCPS } & \text { human-cyber-physical system } \\ \text { AI } & \text { artificial intelligence } \\ \text { human intelligence }\end{array}$

\section{The bigger picture}

Advancing wind turbine technology facilitates the attainment of several United Nations' Sustainable Development Goals (SDGs) by providing affordable and clean energy, fostering innovation for sustainable industrialization, and combating climate change [1]. In the future, the need for WT technology will transcend to a new level in order to support the global demand for reliable and sustainable energy.

Imagine a large-scale renewable energy utility facility - a future offshore wind farm - comprising hundreds of interconnected wind turbines (WTs) each in the deca-Megawatt range. Every single WT of this windfarm resides among the biggest and most complex machines in the world; devised to produce energy consistently for its designated lifetime of 30 years, operating in one of the harshest environments on this planet. Approaching one of these WTs more closely, we identify the rotor blades as the world's largest single 
component made from fiber composite materials which to-date have already exceeded the 100-meter length mark - with a persistent tendency for further growth. The rotor blades constitute one of the most critical components in the wind turbine since they operate in direct interaction with the outside environment, destined to carry the major load acting on the entire WT system.

The fate of a rotor blade is to a considerable degree already determined at the production site where it is born with a set of individual and unique manufacturing defects and imperfections. Upon deployment, during its lifespan, the blade suffers structural deterioration induced by a complex combination of severe dynamic loading, precipitation erosion, lightning strikes, and environmental effects, e.g., temperature fluctuations, humidity, and UV-radiation. The mere fact that individual WT rotor blades exhibit such a stark difference in structural performance during their service life, roots in the diverse evolution of individual manufacturing defects under strongly varying local conditions in the wind farm. It is for this compelling reason that the blades ought to be treated individually while considering every blade's unique history. A direct consequence of treating blades individually is the evocation of a huge parameter space that needs to be controlled efficiently and with confidence to establish workable operation and maintenance (O\&M) schemes.

Current state-of-the-art reactive O\&M strategies for WTs foresee a scheduled maintenance interval of two to three years in addition to unscheduled maintenance operations in situations of critical damage. A reactive O\&M strategy with fixed inspection intervals, on the other hand, is rather costly and amounts to approx. $25 \%-30 \%$ of the Levelized Cost of Energy ( $\mathrm{LCoE})$. Therefore, endeavors are increasingly promoted to shift from reactive- to preventive schemes in order to reduce the cost of O\&M.

The technology for a preventive O\&M strategy is available as a single entity and involves the digital twin (DT) concept developed in recent years. The DT in this work is stipulated as a high-fidelity numerical model that meticulously resembles the physical WT and consequently facilitates accurate predictions of the state of the physical turbine and its components. The idea of a DT dates back to the 1970s where NASA initially implemented this concept in their Apollo space program [2]. After having sunk into oblivion, the DT has seen a renaissance driven by the soaring simultaneous advances in computational speed, numerical modeling techniques, and big data. In more recent works, the concept of a modern DT was outlined for the application to aircraft and aerospace structures [3,4] and further reviews on the application of DT technology can be found in $[5,6]$.

As it is perceived today in the wind energy sector, the prime purpose of a DT is to make accurate predictions of the structural health state of single WT components eventually enabling a reliable, accurate and timely assessment of the entire WT. For this purpose, the DT needs to predict the evolution of the current damage status, considering the prevailing load history, the environmental conditions and manufacturing imperfections among others. A DT, therefore, facilitates a consistent and continuous prediction of the structural performance of individually tagged components from 'the cradle to the grave'. The true power of a DT however, unfolds when coupling it with a structural health monitoring (SHM) system: updating the DT with a live-feed of the as-measured damage state allows predictions of the structural health state with unprecedented accuracy. The integration of DT technology and SHM systems, therefore, opens up the opportunity for more cost-effective scheduling of maintenance intervals.

However, credible projections of wind turbine technology development adumbrate an exponential increase of system complexity, rendering pure human supervised O\&M approaches insufficient and ultimately obsolete. In this situation, it is necessary to introduce more autonomy to the operation of the WT system. Similarly to the autonomous driving technologies [7,8], we envision future WTs having the ability to communicate and operate through machine brains making decisions autonomously in fractions of a second. 
These decisions will not only rely on information from local SHM systems but also depend on supplementary information gathered from other WTs in the wind farm. An individual WT will be 'warned' by other WTs from the fleet about critical incidences such as gusts arriving elsewhere at the wind farm in such a way that mitigation measures can be correctly anticipated and initiated in advance. The role of the DT in such a system is redefined: instead of just aiding human-based decision-making processes, it serves the purpose for training the AI through supervised machine learning. In this context, the role of the human is elevated to a supervisory level. Human intelligence (HI) provides essential inputs to the system to make greater high-level decisions based on perception-driven strategies. In the outlined scenario in this paper, the WT system, the SHM, the DT, the AI, and the HI are fused together into one integral entity that can no longer be considered separately - this system is stipulated in this work as a human-cyber-physical system (HCPS) dedicated for the operation of tomorrows large scale wind farms.

\section{Concept}

WT technology has just arrived in the era of Industry 4.0, which focuses on integrating physical wind turbines and their digital twins into a cyber-physical system. The coupling between digital models and their physical counterparts is facilitated by the rapid development of several technologies namely robust and sophisticated sensors, high-fidelity numerical models, computational power, machine learning, and information transfer technology, e.g. dig data and internet of things (IoT).

In the future, the WT technology will enter a new paradigm in which the diverging strengths of HI and AI are integrated into a single system. An AI conceptually should able to make decisions with human-level precision or even beyond. However, the effectiveness of these decisions depends on the extent and quality of the training the AI received. Quick decision making on an operational routine level is most effectively trained by solving a comprehensive set of numerical problems by using a DT. On the other hand, decision making on a strategic level necessitates the long-term experience and empirical data of the HI gathered over several decades. That is to say, the different ever-evolving WT designs, the development of novel fabrication materials and manufacturing methods, the constantly changing weather conditions and market demands, etc. impose considerable uncertainty that may not be covered by the DT training space of the AI. For this reason, we advocate a semi-autonomous decision-making system in which the training of the AI should rely on a combination of DT technology and HI.

The cooperation between machines and humans is appreciated through human-machine interfaces in order to combine the strategic cognition of humans with nano-quick data processing speed and the consistency of machines. Networked sensor data interoperability enables humans to customize high hierarchy decisions on a strategic level bespoke at scale. The central role of HI is a distinctive feature of Industry 5.0 compared to its predecessor according to researchers [9, 10] and technology visionaries [11]. Inspired by the aforementioned pioneering work, this paper proposes an Industry 5.0 tier concept for the wind energy industry by building on existing Industry 4.0 technologies.

Fig. 1 depicts the concept of the envisioned HCPS for WTs with an operational efficiency far beyond any current schemes. Human-machine cooperation evolves the current DT technology into a semi-autonomous system that uses supervised machine learning to base its decision making process on previous experience and implements the optimal decision in quasi real-time. This goal will be achieved by introducing three feedback loops that work simultaneously and collaboratively.

The red loop in Fig. 1 illustrates that future WTs are embedded in an HCPS and operated by an AI using supervisory control and data acquisition (SCADA) in conjunction with SHM. The AI allows quasi realtime predictions and subsequently determines the optimal operation parameters, which are fed back into the WT controller for instantaneous action. In this feedback process, the AI not only gathers data locally 
from one single WT but also collects far-field data from throughout the wind farm, e.g., approaching wind gusts or WT failure occurring elsewhere in the domain.

The green loop in Fig. 1 depicts the training scheme of the AI, which is based on a continuous update of the damage state in the DT model through a live-feed of SHM measurement data and keeps a 'medical journal' of every individual rotor blade. Subsequently, using the SCADA feed, the DT predicts the evolution of the current damage for a set of key operational parameters to assess structural reliability thereby creating a set of case samples. These case samples are consequently used to train the AI through a continuous recalibration of the parameter space. The computational efficiency of the DT facilitates the solution of a large number of damage evolution problems as a function of different WT control parameters i.e. training samples. The AI will seek decisions that consider the specific preconditions prevalent in the individual blades and avoid certain load cases to decrease the likelihood of failure.

The blue loop in Fig. 1 shows that the HI takes a higher-level supervisory role in direct communication with the AI through a human-machine interface to define the general superordinate directive. The HI will play an essential role in this system by providing innovation to the AI, the DT, and the physical WTs, such as improving and updating the mathematical formations of numerical models used by the DT and, if necessary, to re-align the structural health state with the AI through on-site actions such as damage inspections. The active participation of HI in the HCPS enables a dynamic and creative process that constantly evolves and upgrades itself over time.

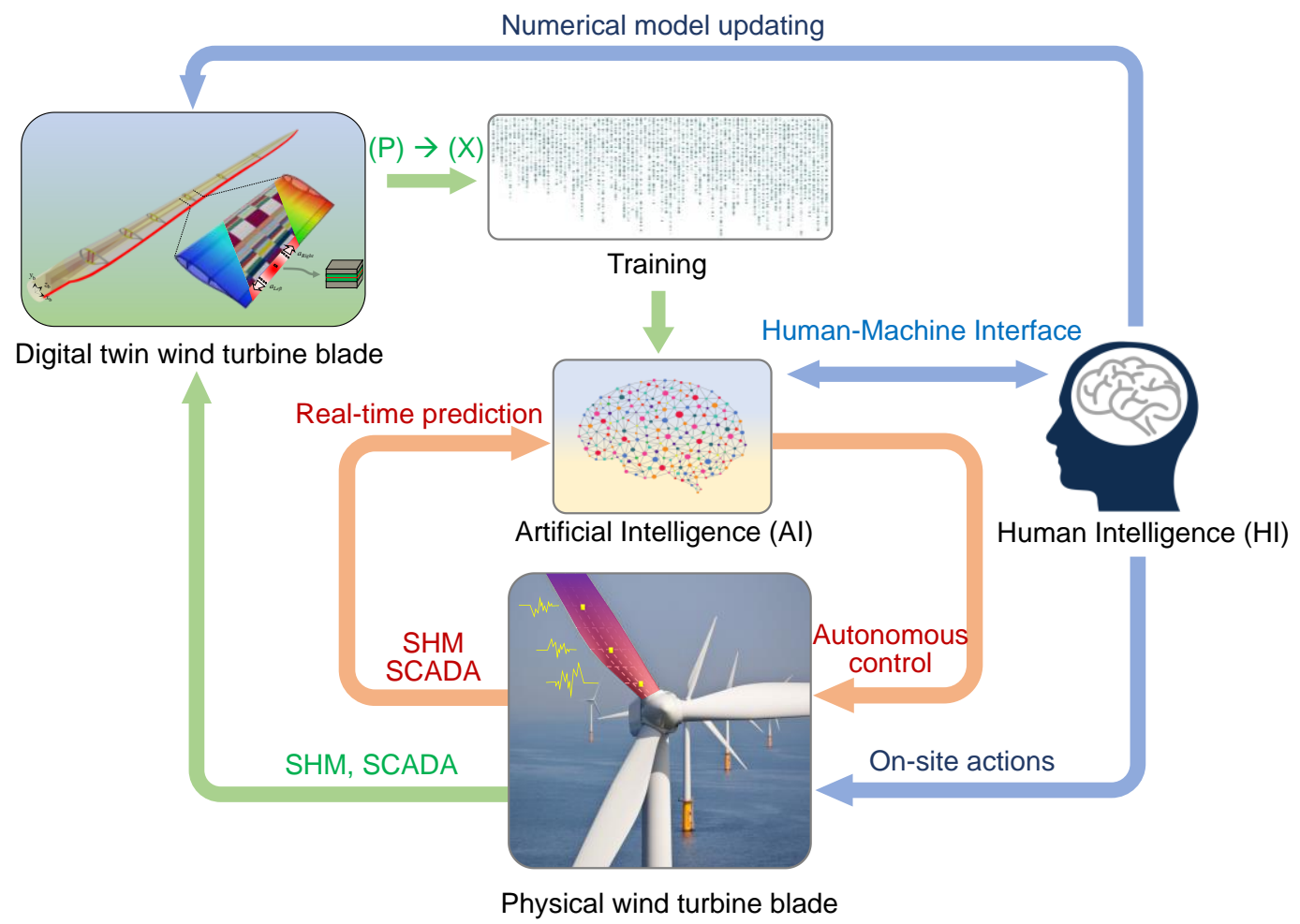

Figure 1. The human-cyber-physical system (HCPS) concept of future wind turbines in the Industry 5.0 era. The system comprises of AI (red loop) that directly controls the operation of the WT in quasi real-time. The AI is trained by a DT that makes predictions that aid the decision-making process. A supervisory human hierarchy is present to provide high-level strategies and perceptiondriven decisions, radical innovations and disruptive technologies to the system that is dynamic, lively and evolving. 
The inner workings of the DT shown in Fig. 1 deserve a closer look and a separate treatise with a particular emphasis on the rotor blades. Fig. 2 shows that the physical blade on the left-hand side is horizontally mapped to a DT on the right-hand side. Communication between the real world and the digital space is established utilizing Industry 4.0 technologies such as IoT, cloud computing, and big data. Three important stages throughout the life cycle of a blade are depicted in Fig. 2 namely (i) manufacturing, (ii) operation and monitoring, and (iii) maintenance. Monitoring of the blade manufacturing process parameters is crucial for the identification and later for the tracking of damage throughout its lifetime. Therefore, the DT already comes into existence with the birth of the blade containing manufacturing defects detected by automatized scanning procedures. The initial defects and manufacturing imperfections are stored in the archive of the DT uniquely associated with the particular blade. A numerical model of the as-built blade is created and the initial defects are discretized. The DT is equipped with a computationally super-efficient probabilitybased numerical damage analysis tool capable of predicting the evolution of the current damage state stored in the archive. After the blade is endowed with SHM sensor systems and installed, it is linked to the DT, which receives a live feed of the structural health status (c.f. Fig. 1). The archive of the DT contains information about the form, size, location, and type of the damage and is continuously updated throughout the blades lifetime utilizing SHM data and blade inspection data.

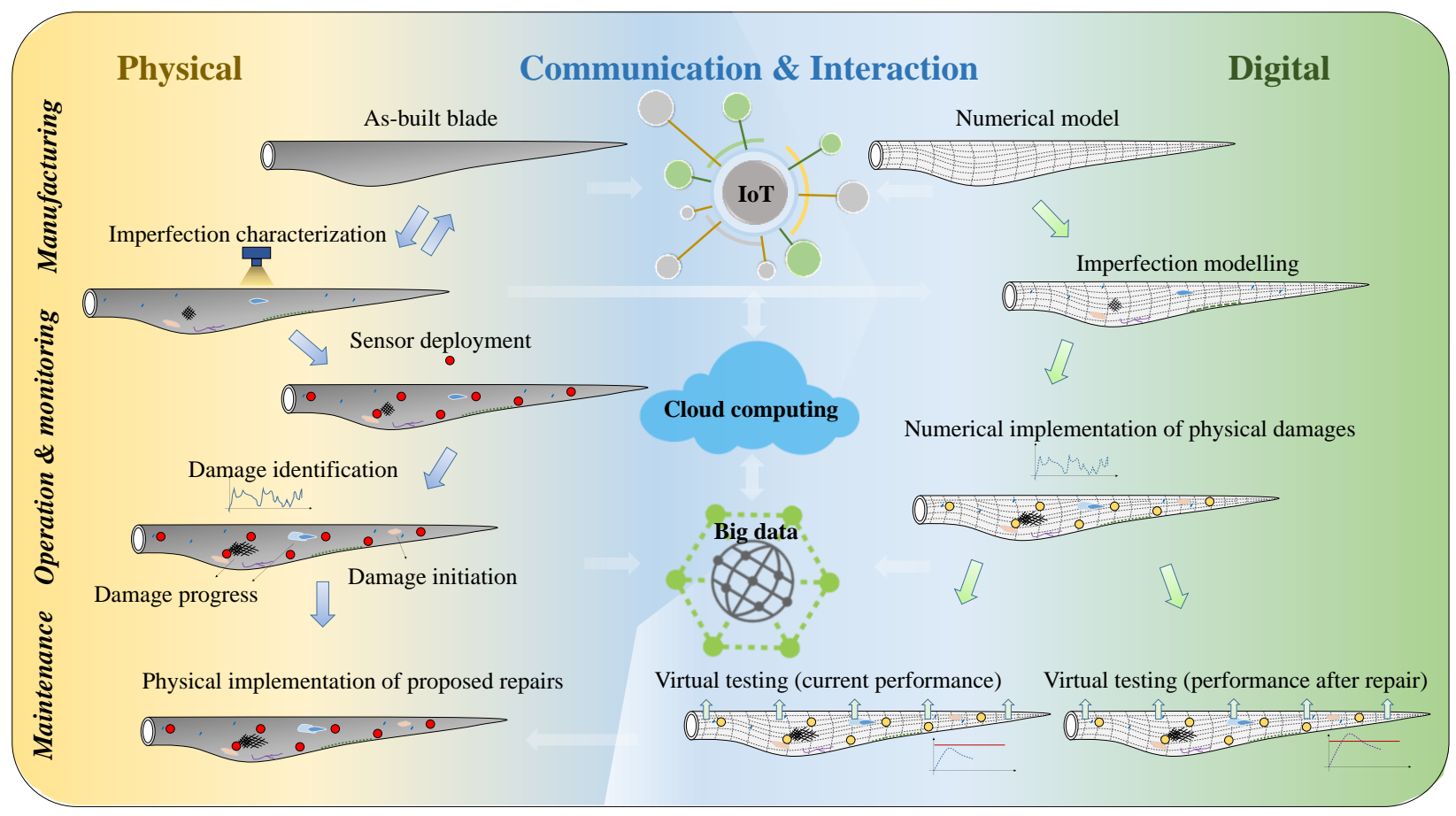

Figure 2. A digital twin follows the entire life cycle of a wind turbine blade from manufacturing, operation, and maintenance. With sensors deployed over the blade, in-service damages are monitored, allowing the numerical model to be continuously updated. The structural integrity can be assessed using the digital twin. Different scenarios can be simulated using virtual testing incorporated with damages, which facilitates reliable and accurate decision-making for operation and maintenance. The physical blade and its digital twin are connected using Industry 4.0 technologies such as the internet of things, cloud computing, big data, etc., forming an integrated cyber-physical system.

\section{Key enabling technologies (KETs)}

As laid out before, technologies like IoT, AI, and machine learning are playing a major role in the HCPS. Complete coverage of all KETs and their interconnectivity involved in such complex systems is beyond the 
scope of this work due to the extensiveness of such an endeavor. Instead, only the KETs deemed to be particularly relevant to WTs and subject to a significant research and development demand are discussed in this section.

The first important KET we want to shed light on pertains to senor technology for SHM. Much like a nervous system continuously passing information of the state of health (e.g. injuries, fatigue) to the brain, the sensors should detect the type, the location, and the size of damages in the different sub-components such as load-carrying spar caps, webs, and lift generating surface panels. Efficient damage detection methods and sensor technologies have to be developed with the ability to cover large areas with reasonable accuracy. Along the lines of medical diagnosis, a suite of complementary sensing methods comprising internal embedded systems and non-destructive remote sensing technologies will be necessary to obtain a sufficiently complete picture of the structural health state. Embedded - in-situ - piezoelectric MEMS sensors, accelerometers, and Bragg refraction based fiber optical sensors reside among the most promising approaches. Ex-situ based SHMs based on measuring light in the visual and infrared spectrum will be implemented through stationary cameras or via autonomous drone inspection without the necessity to interrupt the WT operation. A recent work, Automated QUAlification of DAmages (AQUADA) system [12], uses thermography and computer vision to remotely detect and quantify structural damage below the surface based on the adiabatic heat generated in the material degradation process under cyclic loading. In this quantitative method, both data acquisition and analysis are performed in a single automated step, showing promising results for field applications.

The second important KET is called information translators in this work. In the development of a HCPS, it is crucial to bridge the gap between the physical world such as SHM measurements, and the digital world such as the DT through data interpretation and information translation. First, SHM signals are predominantly an indirect measure of a physical process, which requires a reconstruction method for inverse problems such as e.g., Magnetic Resonance Imaging (MRI) in medical diagnostics. That is, algorithms are necessary to interpret measured data and reconstruct surface cracks, skin/core debonding and delamination from different types of complementary data such as thermal radiation, acoustics, vibration, strains, etc. Second, for the translation of the physical damage into the digital representation, robust and preferably automated tools must be developed. A first step dedicated to performing both interpretation and translation in a single process was taken with AUtomated Damage INspection and Identification from images (AUDIN) [13]. AUDIN utilizes digital image processing technology to obtain the physical characteristics of typical macroscale damages from thermography, ultrasound or shearography and subsequently represents these physical characteristics, e.g., crack size and crack shape, in the finite element model of the DT in an automated way.

The third important KET concerns high-performance numerical damage prediction models. Training of the AI requires the DT to make swift predictions of the damage state evolving in the blade. The stochastic nature of predicting damage evolution in large-scale rotor blades demands probabilistic analysis approaches in which individual simulations are repeated for a variation of the governing parameters. This process craves for computationally super-efficient numerical models that can simulate multiple damage sites under complex loading situations. One of the most notorious bottlenecks in the simulation of damage propagation in large-scale rotor blades - even on supercomputers - is the clash of different characteristic length scales: numerical models must capture damage mechanisms at microscale, i.e., $10^{-4}-10^{-3}$ meters and simultaneously simulate structural response at macroscale, i.e., $10^{1}-10^{2}$ meters. This entails computationally heavy finite element models that render impractical computation times when conventional numerical approaches are adopted. A recently developed method, FASTIGUE [14], is a computationally super-efficient approach dedicated to simulating discrete crack growth in large-scale structures under high-cycle fatigue. FASTIGUE 
gains its efficiency by essentially decoupling the computationally heavy fracture mechanics analysis from the crack growth prediction, contributing to the development of this KET.

The AI will continue to be an important KET playing a central role in the operation of future WTs. Recent advances in AI technology showed promising results in many fields by providing human-level performance. Recent work [15] proved that deep learning approaches can successfully train AI to detect and locate surface damage on rotor blades at approximately $87 \%$ accuracy even for rare damage types from image-based drone inspection. These models illustrated strong performance on the images which are spatially correlated and represent very high dimensional data. For critical condition detection in rotor blades, the AI can deal with low dimensional data, reducing the complexity of the training set, which usually requires thousands of annotated or previously seen examples with relevant indicators. A huge amount of data must be provided for the AI to absorb and learn. By utilizing DT technology in conjunction with numerical methods like FASTIGUE and through extensive measurements from sensor collectives, abundant data can be gathered for the AI to operate effectively.

Another important KET is the human-machine interface enabling the HI to interrupt AI-based decisionmaking by intervening with human intuition in order to break the autonomy when deemed necessary. This is still an open question, however; to some degree solvable by restricting the HI interference actions to only critical conditions based on the confidence level in prediction reported by the AI. The machine learning process is treated as a black box without interpreting the relationship and causality of the features or data points. In order to incorporate a semi-autonomous HCPS, it is crucial to investigate the underlying physics of measurement data and to map the impact on the decision-making process, which is usually beyond a machine's capability. Therefore, the capability of the HI to make expert decisions based on understanding and perception should be incorporated into the AI in order to get the most reliable and accurate results.

\section{Outlook}

The human-cyber-physical system for wind turbines outlined in this article is naturally bound to a rather conceptual level. Projecting Industry 4.0-to-Industry 5.0 technology leaps into the distant future bears a good deal of uncertainty with respect to developmental periods and the precise way of realization. The KETs mentioned in the previous section are just a starting point and have not yet reached the required technological readiness level necessary to sustain a HCPS as outlined in this work. It is important to mention that the relevant KETs in other sectors such as mass production, automotive and aerospace industry are considerably more advanced compared to the wind industry. The advancement in these sectors with extensive efforts carried out by leading high-tech organizations, such as NASA, the U.S. Air Force, Airbus, Tesla, IBM and Huawei, continues to provide a driving force for translating, adapting and implementing similar technologies into the wind energy sector. Technological advancements, particularly in sensor technology, smart materials, drone technology, AI, IoT and quantum computing, continue to grow at an incredible speed - so much so that Industry 5.0 can already be seen on the horizon at the beginning of the third decade of $21^{\text {st }}$ century, see Fig. 3. A HCPS for WTs will become operational and continue to mature in the coming one to two decades, contributing to the United Nations' SDGs in affordable and clean energy, innovation for sustainable industrialization and combating climate change, which will eventually benefit all humanity. 


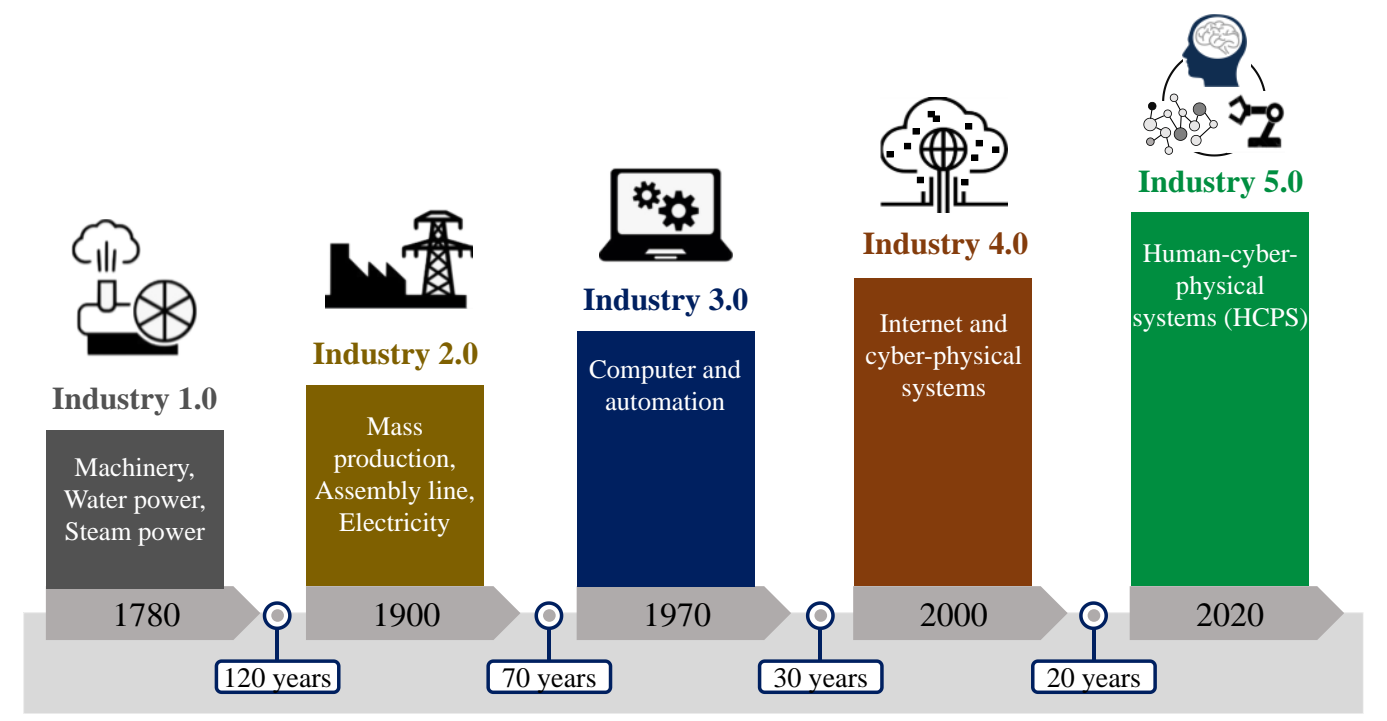

Figure 3. Industrial revolutions in human history. The first industrial revolution used water and steam power to mechanize production. The second industrial revolution used electricity to create mass production. The third industrial revolution used computers to automate production. The on-going fourth industrial revolution uses information technologies to collect the physical world and the digital world. The fifth industrial revolution is expected to bring the human back to the center of operation through a human-cyber-physical system for value creation. Two observations are worth noting. The first one is that the transition time from one industrial revolution to the next becomes considerably shorter, manifesting the rapid development of technologies in the modern era. The second one is that from the second to the fourth industrial revolution, there was a big leap in conceptually new technologies, nevertheless, the next industrial revolution is still based on its predecessor. Projecting from these historical observations suggests a human-cyber-physical system will be the most likely topic of the fifth industrial revolution in the coming one to two decades.

\section{Acknowledgments}

The first two authors gratefully acknowledge the funding received from the RELIABLADE project (Improving Blade Reliability through Application of Digital Twins over Entire Life Cycle, 64018-0068) funded by the Energy Technology Development and Demonstration Program (EUDP) of Denmark.

\section{References}

[1] United Nations, Department of Economic and Social Affairs, The 17 goals. https://sdgs.un.org/goals, retrieved on September 17, 2020.

[2] Lyndon B. Johnson Space Center, National Aeronautics and Space Administration, Apollo program summery report, Houston, Texas, USA, April 1975.

[3] Eric J. Tuegel, Anthony R. Ingraffea, Thomas G. Eason, S. Michael Spottswood, "Reengineering Aircraft Structural Life Prediction Using a Digital Twin", International Journal of Aerospace Engineering, vol. 2011, Article ID 154798, 14 pages, 2011. https://doi.org/10.1155/2011/154798

[4] E. H. Glaessgen, D.S. Stargel, The digital twin paradigm for future NASA and U.S. Air force vehicles, $53^{\text {rd }}$ AIAA/ASME/ASCE/AHS/ASC Structures, Structural Dynamics and Materials Conference, 23 April 2012 26 April 2012, Honolulu, Hawaii, USA. https://doi.org/10.2514/6.2012-1818

[5] F. Tao, H. Zhang, A. Liu and A. Y. C. Nee, "Digital Twin in Industry: State-of-the-Art," in IEEE Transactions on Industrial Informatics, vol. 15, no. 4, pp. 2405-2415, April 2019, doi: 10.1109/TII.2018.2873186.

[6] Fuller, Z. Fan, C. Day and C. Barlow, "Digital Twin: Enabling Technologies, Challenges and Open Research," in IEEE Access, vol. 8, pp. 108952-108971, 2020, doi: 10.1109/ACCESS.2020.2998358. 
[7] S. Mozaffari, O. Y. Al-Jarrah, M. Dianati, P. Jennings and A. Mouzakitis, "Deep Learning-Based Vehicle Behavior Prediction for Autonomous Driving Applications: A Review," in IEEE Transactions on Intelligent Transportation Systems, doi: 10.1109/TITS.2020.3012034.

[8] Umberto Montanaro, Shilp Dixit, Saber Fallah, Mehrdad Dianati, Alan Stevens, David Oxtoby \& Alexandros Mouzakitis (2019) Towards connected autonomous driving: review of use-cases, Vehicle System Dynamics, 57:6, 779-814, DOI: 10.1080/00423114.2018.1492142

[9] Nahavandi S. Industry 5.0—A Human-Centric Solution. Sustainability. 2019; 11(16):4371.

[10] Longo F, Padovano A, Umbrello S. Value-Oriented and Ethical Technology Engineering in Industry 5.0: A Human-Centric Perspective for the Design of the Factory of the Future. Applied Sciences. 2020; 10(12):4182.

[11] E.H. Østergaard, Welcome to Industry 5.0, 2018. https://ww2.isa.org/intech/20180403/, retrieved on September 17, 2020.

[12] Xiao Chen, ASM Shihavuddin, Kenneth Thomsen, Steffen Rasmussen, Kim Branner, AQUADA: Automated Quantification of Damages in Composite Wind Turbine Blades for LCOE Reduction. Wind Energy, to appear. 2020.

[13] Xiao Chen, Sergey Semenov, Automated modeling of damages in large-scale composite structures based on NDT images, $24^{\text {th }}$ International Conference on Composite Structures (ICCS24), University of Porto, Portugal, 14 - 18 June 2021.

[14] Eder, MA \& Chen, X 2020, 'FASTIGUE: A computationally efficient approach for simulating discrete fatigue crack growth in large-scale structures', Engineering Fracture Mechanics, vol. 233, 107075. https://doi.org/10.1016/j.engfracmech.2020.107075

[15] Shihavuddin, ASM, Chen, X, Fedorov, V, Christensen, AN, Riis, NAB, Branner, K, Dahl, AB \& Paulsen, RR 2019, 'Wind Turbine Surface Damage Detection by Deep Learning Aided Drone Inspection Analysis', Energies, vol. 12, no. 4, 676. https://doi.org/10.3390/en12040676 